\title{
Global Imaging of Mitochondrial Morphology in Tissues Using Transgenic Mice Expressing Mitochondrially Targeted Enhanced Green Fluorescent Protein
}

\author{
Hiroshi SHITARA $^{1)}$, Midori SHIMANUKI ${ }^{1,2)}$, \\ Jun-Ichi HAYASHI ${ }^{2)}$, and Hiromichi YONEKAWA ${ }^{1,2)}$ \\ ${ }^{1)}$ Laboratory of Mouse Models for Human Heritable Diseases, The Tokyo Metropolitan Institute of \\ Medical Science, Tokyo 156-8506 and ${ }^{2)}$ Graduate School of Life and Environmental Sciences, \\ University of Tsukuba, Ibaraki 305-8572, Japan
}

\begin{abstract}
The most common approach to analyzing the static morphology of mitochondria involves staining by antibodies or fluorescent dyes specific for mitochondrial components. In this study, we present a new approach using transgenic (Tg) mice, mtGFP-Tg mice, which exclusively express EGFP in the mitochondrial matrix. This Tg strain enables the rapid and easy observation of mitochondria in many kinds of tissues of interest. Recently, many reports have indicated that mitochondrial abnormalities and disease phenotypes are closely associated. mtGFP-Tg mice will be very useful in demonstrating this association, via the use of hybrids of mtGFP-Tg mice and well-established model mice for human diseases.
\end{abstract}

Key words: EGFP, mitochondrial morphology, transgenic mice

Mitochondria play several roles essential for cellular functions, e.g., energy production and signal transduction, and serious diseases can therefore result from deleterious mutations occurring in the mitochondrial genome $[14,16]$. Mitochondrial morphological abnormality has frequently been observed in disease conditions, and several genes controlling mitochondrial dynamics are known to be responsible for neuromuscular degenerative diseases. Therefore, mitochondrial functions are believed to be directly and tightly related to their morphology $[3,10]$. The widely accepted mitochondrial morphology is a bean-shaped form, based on electron microscopy images. However, mitochondria drastically and frequently alter their morphology, repeating dynamic actions such as fusion, fission [15] and transportation [4] in vivo. These morphological changes are thought to be an important part of mitochondrial quality control [13], including mitochondrial complementation [7]. Therefore, the analysis of mitochondrial morphology provides important information, and several procedures for mitochondrial imaging have been proposed and used.

Electron microscopy (EM) and immunoelectron microscopy (IEM) have been widely used to observe mitochondrial morphology and molecular compartmentalization in mitochondria, because only EM is suitable for observing sub-organelle structures. However, there are disadvantages to using EM to observe mitochondrial networks, because EM magnification is not always suitable for observing whole mitochondria or mitochon-

(Received 1 June 2009 / Accepted 10 August 2009)

Address corresponding: H. Shitara, Laboratory of Mouse Models for Human Heritable Diseases, The Tokyo Metropolitan Institute of Medical Science, Tokyo 156-8506, Japan 
drial networks in the cell. Alternative techniques, such as staining with antibodies and/or mitochondria-specific fluorescent dyes, have been developed to observe entire mitochondrial images at the optical microscopic level. However, these techniques also have drawbacks: 1) the techniques require many experimental steps involving complicated, specialized and time-consuming procedures; and 2) ambiguous results, resulting from non-specific immunoreactions or bleaching of the dye, are sometimes observed.

Here we describe a method using a combination of a confocal microscopy and a transgenic mouse strain C57BL/6J-Tg(CAG-Cox8/EGFP)49Rin (common name, mtGFP-Tg), by which mitochondrial morphology can be easily observed as a clear-cut image without any staining procedures. We previously established a transgenic mouse strain in which the mitochondria are labeled with enhanced green fluorescent protein (EGFP) [11, 12]. This strain is available from RIKEN BRC (RBRC02250), Japan. In this strain, as in green mice [9], EGFP cDNA is driven by the CAG promoter, which is known as a strong and ubiquitous expression system [8], and we expected that mitochondria with EGFP fluorescence would be observed in any tissue of interest of mtGFP-Tg mice. Based on the results of co-localization imaging between EGFP and MitoTracker red (Invitrogen), a staining dye specific for mitochondria, EGFP was shown to be exclusively localized in the mitochondria [12]. To prepare tissue sections, mice were perfused with $50 \mathrm{ml}$ of $4 \%$ paraformaldehyde dissolved in PBS (PFA/PBS). Then, the tissues were removed and fixed further in $4 \%$ PFA/PBS overnight at $4{ }^{\circ} \mathrm{C}$. The fixed tissues were introduced into $15 \%$ sucrose dissolved in PBS (sucrose/ PBS) for over $4 \mathrm{~h}$ and then into $30 \%$ sucrose/PBS for over $4 \mathrm{~h}$ at $4^{\circ} \mathrm{C}$. The tissue samples were embedded in Tissue-Tek O.C.T. Compound (Sakura Finetek, Japan) and were frozen in liquid nitrogen. Tissue sections were prepared with a cryostat (Leica) with thicknesses of 10-18 $\mu \mathrm{m}$. The well-dried cryosections were washed with PBS and mounted with SlowFade Gold antifade reagent with 4',6-diamidino-2-phenylindole (DAPI) (Invitrogen). The samples were observed under a confocal laser scanning microscope (LSM510, Zeiss) equipped with Plan Apo 100× objective. The fluorescence activities of DAPI and EGFP were excited with 405 and 488 $\mathrm{nm}$ laser lines, respectively. Fluorescence from DAPI were collected with a longpass filter (LP420) or a bandpass filter (BP420-480), and fluorescence from EGFP were collected with a bandpass filter (BP505-550). Images were analyzed with LSM510 system (Zeiss). All animal experiments were approved by the Institutional Animal Experiment Committee of the Tokyo Metropolitan Institute of Medical Science.

Mitochondria were visualized in the cerebellum, heart, tibialis anterior (TA) muscle, stomach, kidney, pancreas, and liver tissues (Fig. 1), and differences in the fluorescence intensity of the mitochondria was observed among the tissues. In granule cells and Purkinje cells of the cerebellum, mitochondria were visualized as dotted and elongated (Fig. 1A). Fluorescence in the wild-type cerebellum was not observed using the same imaging settings (Fig. 1B); that is, autofluorescence was not detected. In cardiac muscle fibers, numerous large mitochondria were observed to localize in a line along the myofibrils, it is thought this occurs because the heart has high energy requirements, and the EGFP signals were interrupted at the intercalated disks (Figs. 1C and 1D). In skeletal (TA) muscle, localization of smaller mitochondria along the myofibrils was observed (Fig. 1E). In some tissues, mitochondrial sizes varied greatly among the different cell types. In stomach tissue, the mitochondrial size in surface mucus cells was smaller than that in gastric parietal cells (Figs. 1F and 1G). In kidney tissue, the mitochondrial morphology was very different between the convoluted tubules and glomus; that is, larger mitochondria were observed in cells of convoluted tubules whereas smaller mitochondria were observed in cells of the glomus (Fig. 1H). In particular, the length of the mitochondria in the cells of convoluted tubules was longer (up to $4.47 \mu \mathrm{m}$ in this study), and their diameter was $0.434 \mu \mathrm{m}$ ( $\mathrm{SD}=0.099$, Min=0.30, $\operatorname{Max}=0.60, \mathrm{n}=18$ ). Pancreas tissue exhibited mitochondria of the largest size. The mitochondria in acinar cells were much larger than those in islet cells (Fig. 1I), and there was one case in which the width of a mitochondrion was $3.91 \mu \mathrm{m}$ (Fig. 2C). There are many secretion granules in acinar cells, suggesting that the mitochondrial morphology might contribute to the cellular function. These observations were mostly consistent with previous observations [6]. 

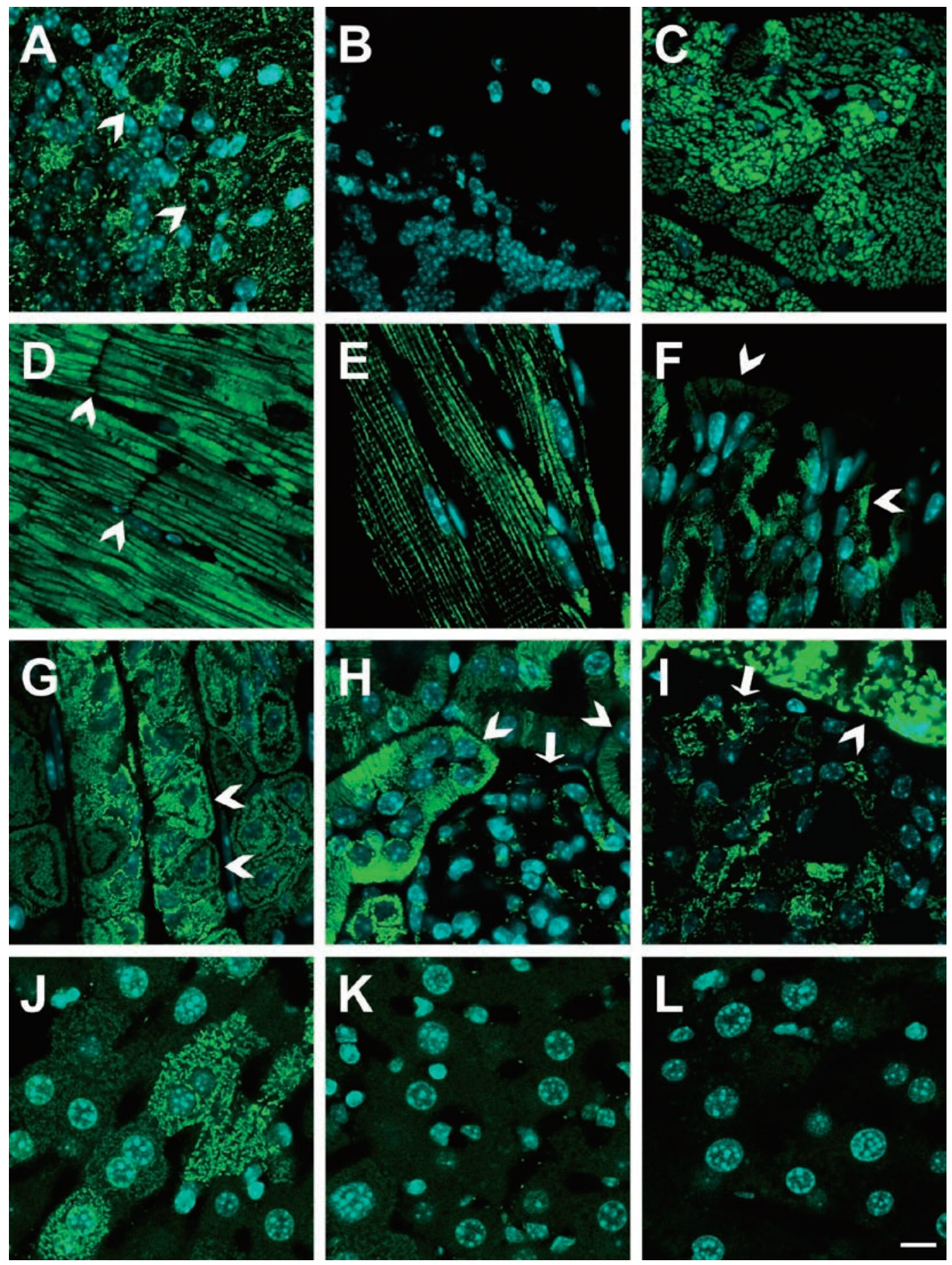

Fig. 1. Mitochondrial morphology in tissues of mtGFP-Tg mice. A one-year-old female Tg mouse was used for analysis. (B) and (L) show tissue images of the wild-type (C57BL/6J) mice, as a negative control. EGFP (green) in mitochondria and DAPI (light blue) in the nuclei were visualized. (A) Border region between the molecular layer and granule cell layer in the cerebellum. Arrowheads show Purkinje cells. (B) Wild-type cerebellum. Fluorescence was not observed using the same imaging setting as in (A). (C) Image of a transverse section from cardiac muscle fiber. (D) Image of a longitudinal section from cardiac muscle fiber. Arrowheads show the region where intercalated disks supposedly lie. (E) Image of a longitudinal section from the tibialis anterior (TA) muscle. (F) The upper part of the gastric mucous layer. Arrowheads show surface mucus cells. (G) The middle part of the gastric mucous layer. Arrowheads show gastric parietal cells. (H) Convoluted tubules (arrowheads) and a glomus (arrow) of the kidney. (I) Acinar cell (arrowhead) and an islet (arrow) of the pancreas. (J) Liver cells. (K) There are regions where fluorescence was not observed in liver cells. (L) Wild-type liver cells. Bar: $10 \mu \mathrm{m}$. 

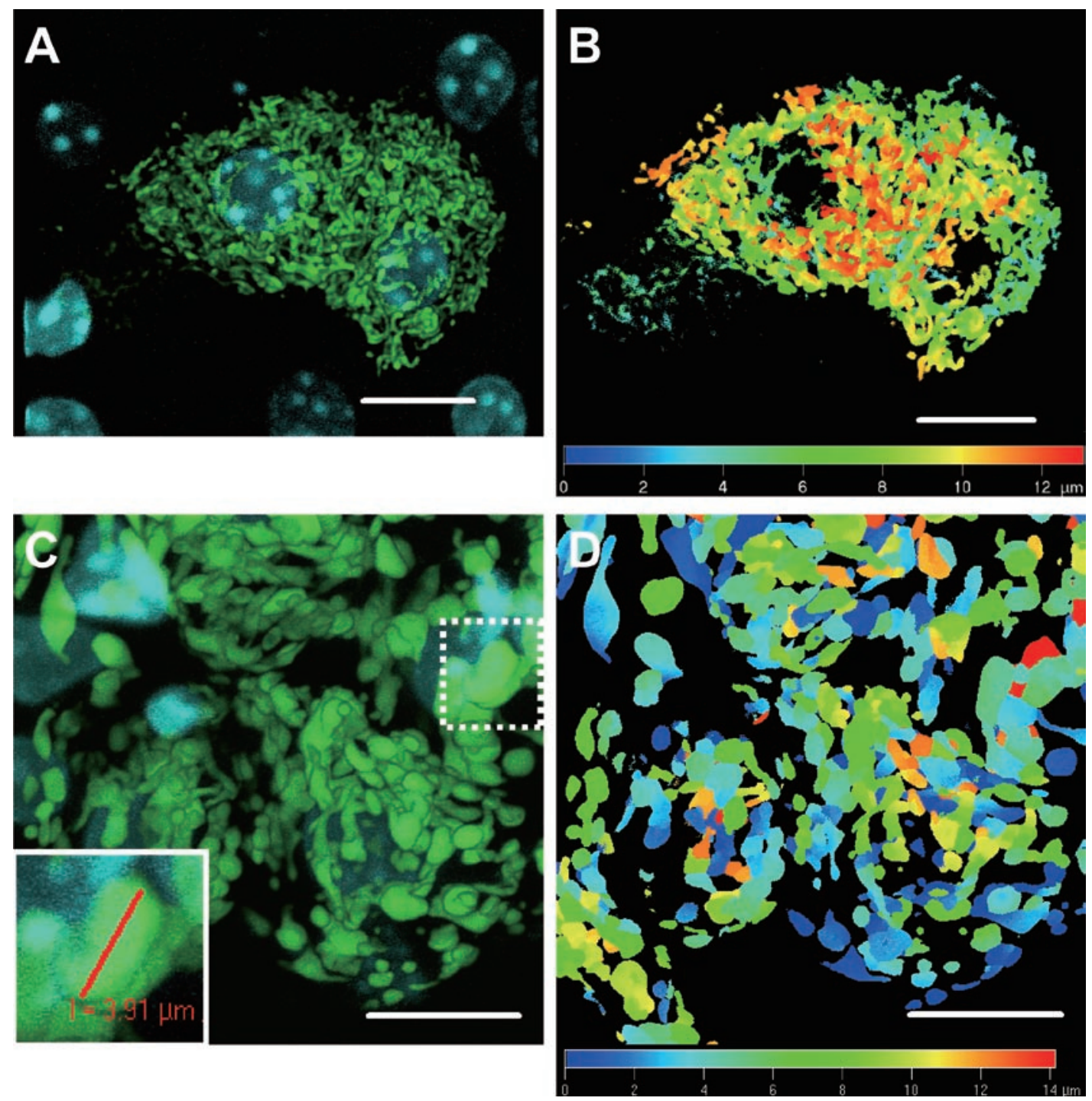

Fig. 2. Three-dimensional images of mitochondrial morphology in tissues of mtGFP-Tg mice. (A) Liver cells. (B) Depth-coding image of (A) with pseudocolor. (C) Acinar cells of the pancreas. Inset shows the magnified figure of the dotted line region. (D) Depth-coding image of (C) with pseudocolor. Bar: $10 \mu \mathrm{m}$.

We also took 3D images to analyze mitochondrial morphology in detail, and large differences in mitochondrial size, morphology, density, and distribution pattern were observed among liver and pancreas tissues. In liver cells, granulous or shorter, "squiggly" mitochondria were observed with a high density (Fig. 2A), and they were localized uniformly throughout the whole cell (Fig. 2B). In pancreatic acinar cells, many large granular mitochondria were visualized (Fig. 2C). Their density was lower than in hepatocytes, but uniform localization of mitochondria was also observed in the acinar cells, as in the liver cells (Fig. 2D).

From the observations described above, it is evident that we can easily analyze mitochondrial morphology in several tissues without complicated procedures. However, it should be noted that several important points should be taken into consideration when using mtGFPTg mice. The EGFP expression level was different among cells even in the same tissue, e.g., some liver cells showed strong fluorescent signals whereas others showed no signal at all (Figs. $1 \mathrm{~J}$ and $1 \mathrm{~K}$ ). This difference in the EGFP intensity in mitochondria may be due to differ- 
ences in EGFP expression among liver cells. This variation in cellular expression is a drawback to the use of mtGFP-Tg mice.

mtGFP-Tg mice provide a useful and promising alternative model for performing mitochondrial morphological analysis, because no time-consuming or specialized experimental procedures are needed, like in conventional methods such as immunohistochemistry or vital staining of mitochondria. Moreover, using a timelapse image acquisition method, mitochondrial dynamics could be observed in primary cultured cells derived from mtGFP-Tg. Recently, many model mice for human diseases associated with mitochondria have been established, $[1,2,5]$. Therefore, the global imaging method we have described should make it very easy to investigate the relationship between the morphological abnormalities of mitochondria and disease phenotypes, because the disease model mice and our mtGFP-Tg mice can be crossed. mtGFP-Tg mice should provide a useful tool for analyzing mitochondria in many fields because mitochondria have many important biological functions.

\section{Acknowledgments}

We would like to thank Dr. L. Cao for valuable comments. This study was supported by a Takeda Science Foundation research grant to H.Y., and partly by Grantsin-Aid from the Japan Society for Promotion of Science and the Ministry of Education, Culture, Sports, Science and Technology of Japan to H.S., J.-I.H., H.Y.

\section{References}

1. Chen, H., Detmer, S.A., Ewald, A.J., Griffin, E.E., Fraser, S.E., and Chan, D.C. 2003. J. Cell Biol. 160: 189-200.

2. Chen, H., McCaffery, J.M., and Chan, D.C. 2007. Cell 130: 548-562.

3. Detmer, S.A. and Chan, D.C. 2007. Nat.Rev. Mol. Cell Biol. 8: 870-879.

4. Hirokawa, N. 1998. Science 279: 519-526.

5. Inoue, K., Nakada, K., Ogura, A., Isobe, K., Goto, Y., Nonaka, I., and Hayashi, J.I. 2000. Nat. Genet. 26: 176181.

6. Krstic, R.V. 1979. Ultrastructure of the Mammalian Cell, Springer-Verlag, NewYork/Berlin.

7. Nakada, K., Inoue, K., Ono, T., Isobe, K., Ogura, A., Goto, Y.I., Nonaka, I., and Hayashi, J.I. 2001 . Nat. Med. 7: 934940.

8. Niwa, H., Yamamura, K., and Miyazaki, J. 1991. Gene 108: 193-199.

9. Okabe, M., Ikawa, M., Kominami, K., Nakanishi, T., and Nishimune, Y. 1997. FEBS Lett. 407: 313-319.

10. Parone, P.A., Da Cruz, S., Tondera, D., Mattenberger, Y., James, D.I., Maechler, P., Barja, F., and Martinou, J.C. 2008. PLOS ONE 3: e3257.

11. Rizzuto, R., Brini, M., Pizzo, P., Murgia, M., and Pozzan, T. 1995. Curr. Biol. 5: 635-642.

12. Shitara, H., Kaneda, H., Sato, A., Iwasaki, K., Hayashi, J., Taya, C., and Yonekawa, H. 2001. FEBS Lett. 500: 7-11.

13. Tatsuta, T. and Langer, T. 2008. EMBO J. 27: 306-314.

14. Wallace, D.C. 1999. Science 283: 1482-1488.

15. Yaffe, M.P. 1999. Nat. Cell Biol. 1: E149-150.

16. Yonekawa, H. and Akita, Y. 2008. FEBS J. 275: 40054013. 